\title{
Hang in There: A Novel Body-Centric Interactive Playground
}

\author{
Robby van Delden ${ }^{1}$, Alejandro Moreno ${ }^{1}$, Carlos Ramos ${ }^{2}$, Gonçalo Carrasco ${ }^{2}$, \\ Dennis Reidsma ${ }^{1}$, and Ronald Poppe ${ }^{1}$ \\ 1 Human Media Interaction, University of Twente, \\ P.O. Box 217, Enschede, The Netherlands \\ \{r.w.vandelden, a.m.moreno, d.reidsma, r.w.poppe\}@utwente.nl \\ ${ }^{2}$ Faculdade de Ciências e Tecnologia, Universidade Nova de Lisboa, \\ 2829-516 Caparica, Portugal \\ \{carlosjmramos, gbcarrasco\}@gmail.com
}

\begin{abstract}
We introduce and evaluate a first version of a novel bodycentric playground that aims at increasing bodily exertion and immersion. The concept centers around the player, who is suspended from the ceiling using a rope and climbing harness and stands on a tilted platform. This caused players to assume a body posture that elicits the feeling of flying, which was further enhanced by the flying game that they played. We discuss the choices made in the type of body movements, and how these relate to different aspects such as movement mimicry and exertion. We performed a user study, in which the hanging position was compared to a setting where players stood on the ground. We found no significant differences in the amount of movement and perceived engagement between the two conditions. However, there was a tendency of favoring the hanging position. Moreover, we observed that the placement of game elements affected the movement patterns.
\end{abstract}

Keywords: Body-centric, interactive playground, engagement, exertion, postures.

\section{Introduction}

Computer entertainment taps into a variety of the prerequisites to feel good in life [21. At the same time, there are growing health concerns because these advances in technology have led children to adopt a sedentary behavior, causing an increase in obesity 22. Nonetheless, the introduction of the very technology that caused this issue can also be used to counter it, effectively turning the problem into a solution [7. Researchers have begun to study how to create interactive playgrounds, interactive installations equipped with sensors and actuators where children are encouraged to exercise and interact socially through engaging game experiences [19:20 9]. These interactive playgrounds are co-located interactive spaces, ranging from a few square meters to public squares equipped with a 
range of sensors and feedback devices. The advent of affordable, robust technologies such as Microsoft Kinect, Arduino, projections and LEDs greatly facilitates the implementation of such playgrounds.

Studies have shown that making the body the center of the experience in these installations leads to higher engagement and immersion [2] and promotes social interaction [8]. This greatly attributes to the success of games that use bodily interaction, e.g. using Nintendo Wii-motes and Microsoft's Kinect. Often, the gameplay of these games is straightforward. However, the link between engagement and bodily exertion is bi-directional: games that provide an engaging gameplay also encourage people to move more [5]1. This increases their immersion in the game, thus creating a feedback loop where gameplay, engagement and physical exertion play key roles [1. Given that these three elements are strongly related, it makes sense to actively consider them in the design of body-centric interactive installations.

Pasch et al. [15] identify four aspects that relate players' movements to their immersion and engagement levels: natural control, mimicry of movement, proprioceptive feedback and physical challenge. Each of these have informed the creation of the first prototype of Hang in There, a novel body-centric interactive playground (See Figures 1 and 2 for an impression). In this paper, we describe and evaluate the playground, which is aimed at promoting physical activity by providing a new user experience that focuses on creating an increased sense of immersion. A virtual 3D world with a digital version of the player is shown on a large screen in front of the player. The aim of the game is to fly through this world and collect as many coins and power-ups, while avoiding obstacles and power-downs. The game is controlled by full-body movements. To provide a sensory stimulus of flying, the player is suspended using a climbing harness and rope while standing on a 45-degrees tilted platform.

We present an overview on postures, physical exertion and engagement in games and simulations in the next section. The design, setup and implementation of the Hang in There installation is discussed in Section 3 , followed by a user study to investigate the difference in engagement between playing the game in a standing and hanging position. We conclude with a discussion on our findings and present avenues of future work.

\section{Exertion, Postures and Engagement}

Several studies have shown that body-centric control in games increase the engagement and immersion of the user (see [114 for an overview). Furthermore, Riskind and Gotay showed that putting someone in a certain posture can have a effect on their level of task persistence, expectations and (self-)perceived emotional state [17. Bianchi-Berthouze et al. [2] tested how playing Guitar Hero with a standard PlayStation controller and a guitar-like controller affected the engagement of the player. They found that a more natural way of control encourages the player to immerse himself in the game, which leads to a greater emotional experience. In another study with Guitar Hero they instructed users 


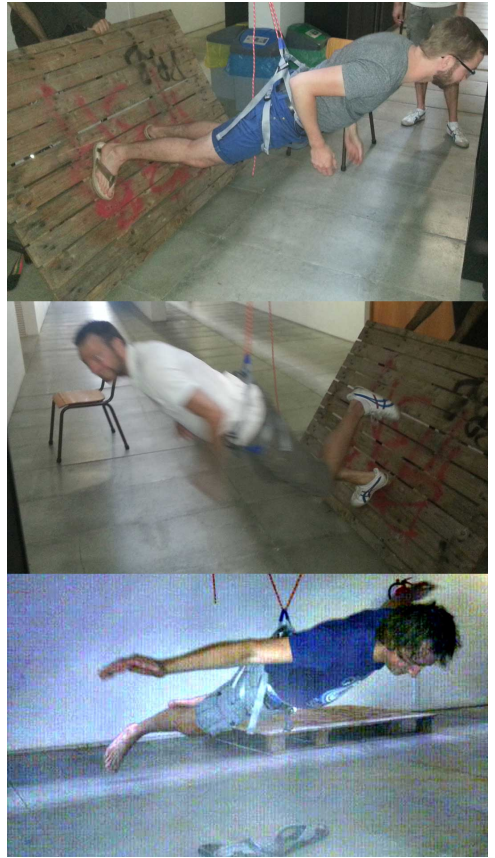

Fig. 1. Exploration of positions

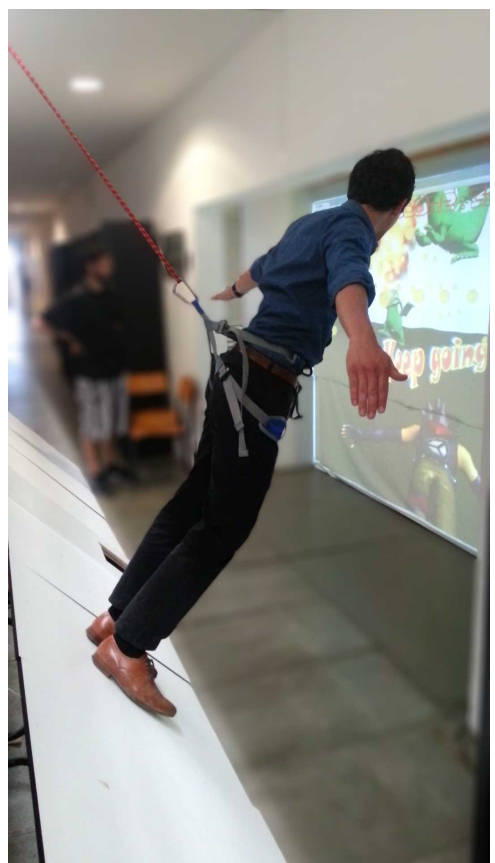

Fig. 2. The final prototype

about the ability to exhibit a more expressive movement and posture, tilting the guitar instead of pushing a button. This led to a different type of engagement and play, which tended to change to a more fantasy rich and more emerged play 2]. Based on these and other studies Bianchi-Berthouze concluded that affording body movements linked to role-play resulted in higher player engagement and presence in the virtual world, users becoming more socially and probably more emotionally involved [1].

Making use of affording posture change has been done in some commercial games, simulators and art installations. There are virtual glider games in which users hang in a glider in order to fly in a virtual environment, such as the Dream Glider by Dreamilty Technologies, "Virtual Hang-Gliding over Rio de Janeiro" by Soares et al. that even included a fan for simulating the wind [18, and another "Virtual hang gliding over Rio" by YDreams for Rock in Rio 2011. There are also several flight simulators that tilt the user, including the Jetpack Simulator by Martin Aircraft Company. In this training simulator a user is strapped on a small tilting platform that rotates slightly above the floor with the center point of rotation around hip height. With respect to sports there are also simulators which allow for realistic postures and movement that make the user more engaged and primarily attempt to train the user efficiently. For instance, Skytechsport created the Alpine Racer downhill simulator, in which the user experiences forces including vibrations and tilt, limited to a flat horizontal plane, that simulate real skies and slopes. Ars Electronica Futurelab created Humphrey (II), an interactive 
body-centric flight simulator. The user was hanging in a suit that is suspended from the ceiling with ropes. Furthermore, they implemented a force feedback system to simulate the forces during flight. The user could either fly through the city of Linz or dive in the Danube by making body movements 1 Fels et al. exhibited an installation that allowed users to swim while being in the air [4. The participant was suspended in a harness that was suspended from a frame with several ropes. In the 1990s Sega created a rotating arcade cabinet called the R360. The user was strapped onto the cabinet's chair similar as is done in a roller-coaster. It had two rotating axes allowing for full rotation, the users could thus even be upside down. Pasch et al. [15] studied how movement in sports games affected the game experience using the Nintendo Wii. They observed that play styles changed according to the motivation or goal of the players, namely if they were playing to relax or to compete. They also found that control is an important factor in the experience. By observing and interviewing the players, they were able to define four movement features that affect players' immersion: natural control, mimicry of movements, proprioceptive feedback and physical challenge. Natural control takes into account how similar the movements of the player are with respect to those they would perform in real life. Mimicry of movement refers to the mapping of the players' movements to those of their avatars or, if there is no avatar, in-game movements. Proprioceptive feedback encompasses the sensory input. Lastly, physical challenge refers to how much exertion the game requires. Two of the main goals of interactive playgrounds are providing engaging, fun experiences and encouraging physical activity [16. Therefore, these four features are specially important in the design of bodycentric interactive installations since they link engagement and immersion to a player's movement and, by consequence, to their physical activity.

Yao et al. 23. built a tangible "RopePlus" installation in which people could remotely interact and play two games: "Multi-Fly" and "Multi-Jump". The main element of the system was a rope connected to a motor which sensed and limited the movement of the rope. In its handle, the rope had an accelerometer and a wireless communicator. When any of the two games was projected, the player could either fly a kite or play jump the rope by using the rope that was provided by the system. This is a typical example of natural control, as the movement are similar as those performed with a real kite, or when jumping rope on a traditional playground. Another example is the "Kick Ass Kung Fu" installation designed by Hämäläinen et al. [6]. They designed a martial arts game where a player could fight AI or player-controlled virtual characters. The setup was composed a of a cushioned arena, with one big screen located at each side, where the player was allowed to move. A webcam was used to observe the player who was, after some computer vision processing, inserted into the game as one of the fighters. On these screens, a standard 2D fighting platform game was projected, and the player could move from side to side while punching, kicking, jumping or doing

\footnotetext{
${ }^{1}$ A more detailed description of the Humprey II can be found on http://90.146.8.18/en/archives/picture_ausgabe_03_new. asp?iAreaID=294\& showAreaID $=294 \&$ i ImageID $=44618$
} 
acrobatic moves to attack the enemy. Again, these movements are a natural way of control, and they are mimicked in the virtual world. As the movements were exagerated in the virtual world, the player was persuaded to make even more spectacular jumps and kicks.

Recently, a lot of attention has been given to installations that focus on the physical challenge feature. Mueller introduced the term exertion interfaces for games that require the player to undergo physical exertion [10. He argued that this component makes the game more fun and encourages social bonding. For instance, Mueller et al. [12] designed a game of table tennis for three participants. The setup was similar to that which one uses when playing alone, i.e. instead of a net, a wall is located in the center of the table so the ball bounces back to the player when he volleys. The other players were back-projected to this wall, each one occupying half of the wall. The players competed against each other by breaking blocks that were overlaid on the video feed. They found the participants engaged in social interactions often and enjoyed the competition element, even though they sometimes got exhausted.

Although exertion games are able to increase a player's engagement and immersion levels, there is also an important relationship between gameplay and the willingness to exercise. In their "Astrojumper" game, Finkelstein et al. [5] found that gameplay attractiveness was related to exercise effectiveness. They developed a virtual reality game where players had to dodge virtual planets that sped towards them. The players wore stereoscopic goggles along with trackers on their bodies to track their movements. These trackers gave the players a lot of freedom in their movements, as they could move to the sides, jump, duck, and shoot lasers to destroy objects. At the end of the playing session, the participants stated they enjoyed the game and were extremely motivated to play again, demonstrating that attractive gameplay can encourage physical activity. Similar observations were made for the "Hanging off a Bar" installation [13]. The setup consisted of an exercise bar that players were to hang onto while a game was projected underneath them. The game consists of a flowing river where, occasionally, a raft drifts down and the player is allowed to let go of the bar. However, once the raft drifts off the player has to hang onto the bar again. The player loses if he lets go of the bar and "falls" into the water. In the evaluation phase, players mentioned that they would invest more physically if, among other things, the game facilitated fearful emotions or allowed to play with other people, both of which make up elements of gameplay.

\section{Hang in There System Design}

The different movement features and their relation to engagement must be considered when designing body-centric interfaces. In this section, we discuss how we designed an engaging installation that supports proprioceptive feedback, natural control, movement mimicking and physical exertion, at the same time. By carefully designing the different game elements, we aim at increasing immersion and engagement, with the ultimate goal of increasing the fun experience and the amount of movement. 


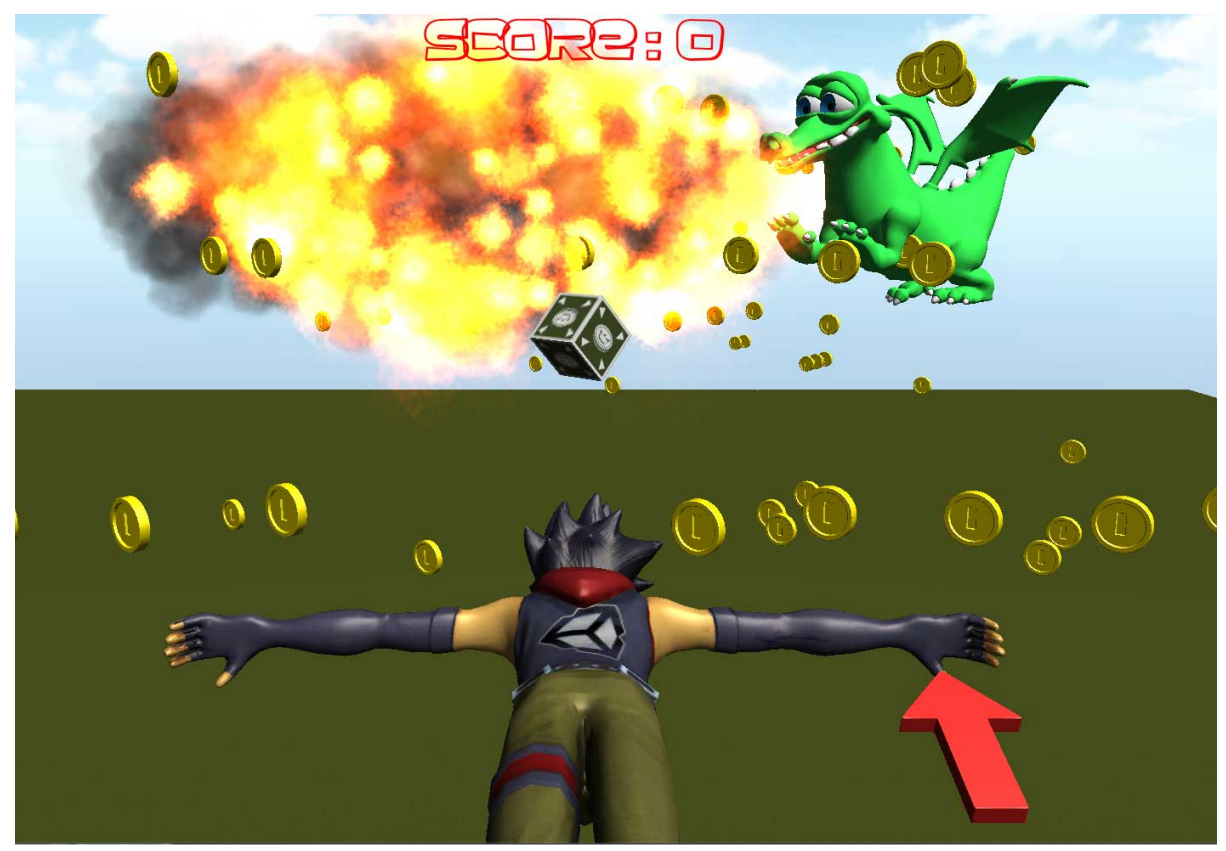

Fig. 3. Screenshot of gameplay with avatar in starting position

\subsection{Physical Setup}

We set out to design a novel body-centric interactive playground that elicits the experience of flying in the user. This feeling is created by putting players in a extreme forward tilted position. As a consequence, the center of gravity is in front of the feet, forcing the body to fall forward. To accomplish this, the player stands on a smooth, tilted platform while being attached to a climbing rope and harness (Figure 2). Different tilt angles for the platform were tested (Figure 1) to experience the feeling of suspension, and the comfort of the position and equipment. Being totally suspended provided a great experience, however it was unfeasible to maintain this position for long because of the pain caused by the chosen equipment. This could be remedied by adding more support points at the cost of a reduced feeling of freedom. Even with the current setup, there is a certain level of discomfort, especially around the hips and abdomen, that limits the maximum amount of playtime. Better/different equipment could be tried in the future.

\subsection{System Architecture}

We had a single system consisting of a game engine with a Kinect for Xbox sensor running on a single laptop, outputted to a projector and speakers. The game is created in Unity 4.1, a free to use $3 \mathrm{D}$ game development system and engine. To 
interpret and deal with the movements of the user, we make use of Kinect for Windows SDK 1.6 and an existing Kinect wrapper from the asset store of Unity $3 \mathrm{D}^{2}$. To allow for reasonable performance and ease of use we altered some parts of this wrapper. We removed the automatic rotation of the Kinect sensor and the gesture recognition functionality.

\subsection{Game Controls}

The game consists of a player flying through a virtual world, in which he is represented by a 3D avatar in third-person view (see Figure 3). The world is projected on a wall in front of the player. Once a player is recognized with the Kinect skeleton tracker, the avatar will move forward at a constant speed. The game lasts 109 seconds, and ends with the player going through the finish line, since the speed of the player is unaffected by any game interaction.

The player's movements are mimicked by the avatar and are used to navigate through the virtual world (see Figure 4). Specifically, the player can navigate to the left or right of the virtual world by moving to his left or right respectively. This movement is physically demanding because his center of gravity is in front of him, but also because the player is drawn back to the center because of the harness attachment point. Therefore, maintaining a steady position on any one side requires the player to use muscles located in the legs and abdomen. The player can also ascend, descend or maintain his current altitude in the virtual world by quickly flapping his arms, holding them close to his body, or extending them outwards respectively. These movements exercise the arms and shoulders.

The Kinect is placed on the floor pointing towards the person hanging on the platform. In this set-up, the legs were not always recognized properly, among other reasons due to the short distance between the player and the Kinect. Therefore, we decided to remove the bindings of those "Kinect joints" to the avatar. Instead, the legs simply followed the rotation of the hip bone, always fully extended.

We evidenced that the instinctive way to flap the arms changed from player to player, but it also depended on their current position. When hanging, most players moved their arms up and down along their body, while others moved them back and forth, as if they were birds. In the standing position, almost every player moved their arms up and down along their bodies. Since the platform where players stand on is tilted approximately 45 degrees, we allowed users to flap either up and down, or back and forth. The elbow joints gave the best recognition results for these movements. Hands, for instance, were sometimes not recognized if they were too close to the body or too far to the sides for the Kinect to recognize.

The recognition of flapping is done with the displacement in y-axis (vertical) and z-axis (depth perpendicular to Kinect) of the elbow joints. This displacement is normalized with the length of the hips to the shoulders, to even out differences

\footnotetext{
${ }^{2}$ Kinect with $M S-S D K$, version 1.3, https://www.assetstore.unity3d.com/ \#/content/7747
} 


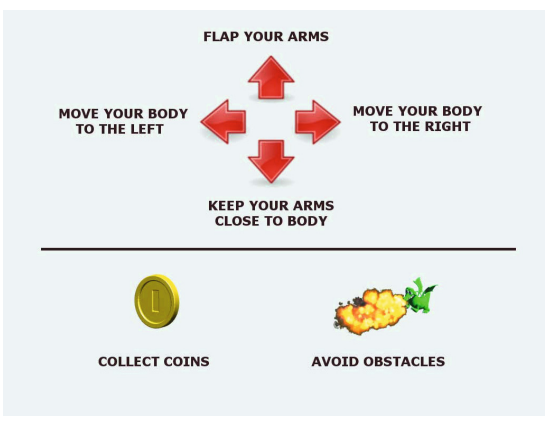

Fig. 4. Game instructions

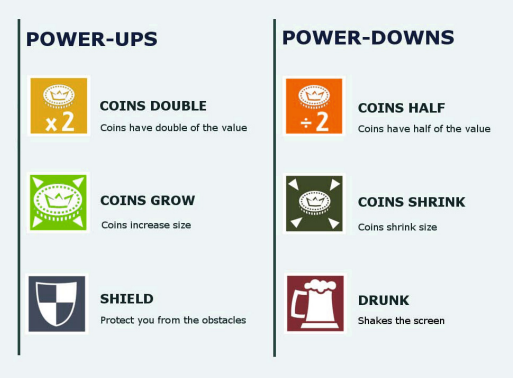

Fig. 5. Power-ups and downs

in body lengths between subjects. If the sum of displacement over the last 12 frames (approximately 0,5 second) is higher than a predetermined threshold, the avatar moves up with a force proportional to this displacement.Falling, or descending, occurs when the player keeps his arms close to the body. The vertical distance between the elbows and hips are normalized based on the distance between the hips and shoulders. If this normalized distance is below a certain threshold, the avatar will descend with a force proportional to this value. These forces should allow the user to have some control over the vertical movement.

Even though we wanted the players to use their whole body to play, the fact that the Kinect could not always recover the location of the legs was an inconvenience. We circumvented this by looking at the absolute position of the player's hip joint in space. By stepping left and right, this joint moves and we use this to position the player's avatar. We tested several multiplication values to empirically find one that allowed the users to move comfortably and fairly, while exaggerating the movements slightly to give them a sense of empowerment.

\subsection{Game Objects}

The goal of the game is to collect as many points as possible by collecting coins that float in the air. However, there are obstacles represented as fire spitting dragon: 3 , that will reduce the number of points. To persuade the players to move more to the sides, which should require more physical effort, more coins are placed to the sides of the virtual world and more dragons in the middle. In fact, we placed more coins at the right side of the screen, compared to the left side, to be able to measure whether coin placement would affect the players' sideways movements.

Furthermore, there are several power-ups that make it easier to collect points and power-downs that make it harder to do so (see Figure 5). These modifiers, which are represented as textured boxes, are placed throughout the virtual world to make the game more engaging and add some variation into the gameplay.

\footnotetext{
${ }^{3}$ Dragon model Larry obtained from http://www.sharecg.com/v/37318/
} 
There are three classes of modifiers in overall, with each class having a power-up and a power-down. The first class affects the amount of coins the player collects ("coins double", "coins half"), the second affects the size of the coins present in the field ("coins grow", "coins shrink"), and the third affects the context of the game ("shield", "drunk"). There are 8 instances (4 power-ups and 4 powerdowns) of each class scattered in the game, e.g. 4 modifiers to double the collected coins and 4 modifiers to halve the collected coins. The only exception is the contextual modifier class, which had 8 power-downs and 4 power-ups. The first two classes are self-explanatory, however the contextual modifiers warrant an explanation. The "drunk" power-down severely shakes the screen of the player when collided against, making it harder to collect coins for a brief period of time. The number of instances of this power-down was doubled because it had a high entertaining value during the development of the installation. The other contextual modifier, the "shield" power-up, prevents the player from losing coins when colliding against dragons (or their fire) once. The duration of the shield is short, and disappears if not used. The shield is represented as a purple, semitransparent half-sphere that directly in front of the player.

Multimodal feedback is provided during the game when different events occur. The player's score is shown in the top center of the screen to encourage him to collect as many coins as possible. Encouraging messages, along with appropriate sounds, are also triggered when players collect power ups. When hitting the dragon or its fire, the camera shakes and a short burning sound is played. Every collected coin triggers a "Mario Bros" coin collecting sound. When the avatar of the user is flying too low or too high, a warning sign is shown. When the players reaches certain milestone scores, he is praised with "Awesome!", "Great!" or "Keep going!" messages. Upon crossing the finish line, a firework sequence is shown and a cheering sound is played.

\section{User Study}

We wanted to evaluate whether being suspended elicited a sense of immersion and increased the engagement of the Hang in There installation. To do this, we tested two different conditions in which the players played the game while standing or in the tilted hanging position. The design, physical setup, procedure and results are discussed in this section.

\subsection{Experimental Design}

In order to compare the standing and suspended conditions, the game and the movements to control the avatar were kept equal. Therefore, only the physical position of the body changed. We used a within-subjects design, so each participant played the same game once in both physical positions. The order was alternated between sessions. There is no standard method to evaluate exertion interfaces 11. Here, we use questionnaires, an informal interview, and an analysis of the movements of the players. 


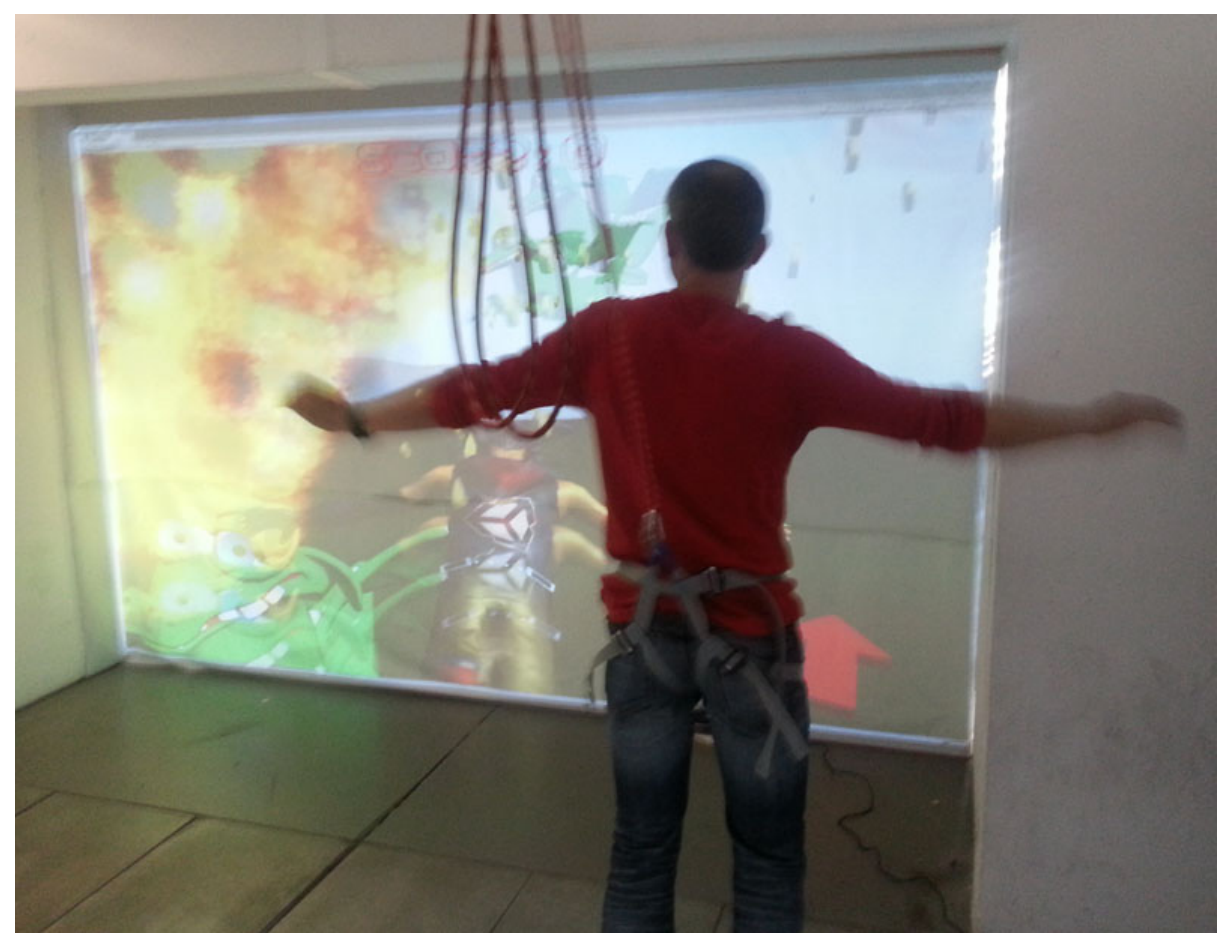

Fig. 6. Standing condition of Hang in There

\subsection{Physical Setup}

We conducted the experiments at the Unversidade Nova de Lisboa. The setup was placed in a corridor of the main building of the faculty of science and technology. The physical setup for the experiment consisted of a tilted platform, a harness, a climbing rope, a projector, a laptop, a projection surface and a Kinect. The tilted platform was built with wooden boards that were fixed together and supported by table frames with several counterweights. The slope was about 45 degrees. In the hanging position, the participant stood on the tilted platform, held by the harness. The player faced the screen in a tilted position, assuming a body posture that elicits the feeling of flying. In the standing condition, the participant assumed an upward position, parallel to the screen. During the standing condition, the player was still held with the harness to limit the motion and keep the amplitude of movements from left to right similar in both conditions (Figure 6). Again, the participant faced the screen.

The projector was placed on the floor, behind the platform, by cutting a hole in it. This was done to maximize the projection area, while taking care to avoid casting shadows. A $3 \times 2$-meter projection cloth was fixed to a wall, about two meters from the platform. The large dimensions of the screen were thought to 
facilitate the feeling of immersion in the game (see Figure 6). The Kinect camera was placed on the floor, just in front of the cloth.

\subsection{Procedure}

After signing a consent form, the harness was put on and the rules and control of the game were briefly explained. The participant had up to two minutes to test run the game and familiarize himself with the movements and controls. The test run was played while the participant was standing on the floor. Afterwards, the first game was played, in a hanging or standing position, depending on the specified order. Immediately after playing the game, the participant filled in an online version of the Gaming Engagement Questionnaire (GEQ Revised) [3]. In our revised version of the GEQ questionnaire, items that did not relate to the concept of our game were excluded and some of the terminology was adapted to our installation (see Appendix $\mathrm{A}$ for the revised questionnaire). Following this, the participant returned to the platform to play again in the second condition. Once finished, the participant again completed the GEQ. Finally, we asked brief questions about the game experience. For each participant, the duration of the entire experiment was around 20 minutes. In addition to the questionnaires and interview, we recorded the joint data of the Kinect skeleton tracker. These joint positions and rotations were saved every 6 frames.

\subsection{Results}

Fourteen participants played the game (11 men, 3 women, average age: 30.2 years, age range: 24-44 years). They were researchers and students of different fields, including computer science and psychology. Each participant played the game twice, once in each condition.

GEQ-R. We excluded the question "How often do you play games from this genre?" of the revised GEQ as it does not measure engagement. The results for questions $17,18,21$ and 22 were mirrored in order to have a positive value corresponding to an increase in engagement for all questions. We first checked the correlation between the items, which resulted in a Cronbach's alpha of .843 if both sessions are included. This suggests that the questionnaire successfully measures a scale for engagement. Although the mean engagement value for the GEQ-r was slightly higher for the hanging $(\mu=4.91, \sigma=0.65)$ compared to the standing position $(\mu=4.77, \sigma=0.64)$ a paired-sample two-tailed t-test did not show significant difference between the two conditions.

Next, we investigate some questions into more detail. The majority of the players $(n=8)$ answered they were more inclined to play the game again for the hanging condition. The other six participants gave the same response for both conditions $(\mu=5.79, \sigma=0.97$ for hanging and $\mu=4.79, \sigma=1.53$ for standing). A paired sample t-test indicated a significant difference $(p<0.01)$. When corrected for the number of questions, to eliminate the probability of obtaining significant differences by chance, we divided $\alpha$ by the number of questions 

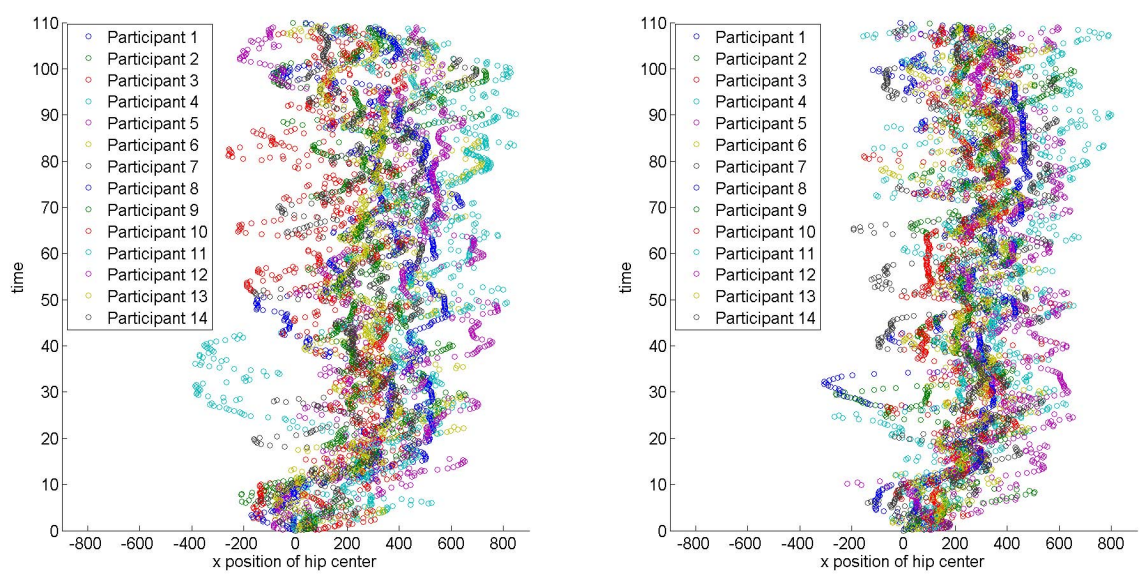

Fig. 7. Scatter plot for standing (left) and hanging condition (right), representing hip position on the $\mathrm{x}$-axis in $\mathrm{mm}$

and found that the difference was not significant. We found marginal differences for questions Q2 ("How much delay did you experience") $(p=0.06)$ and Q20 ("How completely were you engaged in the game") $(p=0.07)$, indicating a favorable trend towards the hanging condition. Other questions did not approach significance level.

Movement Logs. Next, we turn to the tracked joint positions. We noticed that the position of the extremities (hands and feet) were not always estimated. In the game, we therefore used the position of the elbows to detect the flapping. Moreover, we did not use the positions of the knees and feet, but rather the estimated hip positions. In this analysis, we take a similar approach.

We start by analyzing the variation in the hip center in the direction paralel to the screen. Overall, results showed no difference between both conditions in terms of movement in this direction. Since one of our goals was to persuade people to move to the sides instead of staying in the center, we analyzed the position of the avatar. To do this, we needed to compensate for the start position, since the data showed that people started, on average, $2 \mathrm{~cm}$ (standing) and $4 \mathrm{~cm}$ (hanging) to the right. The average position of the players was significantly more to the sides than the center, as measured with a one-sample two-sided t-test $(p<0.001)$, with the average hip position on the x-axis over both conditions per participant. We found a systematic bias to the right of $28 \mathrm{~cm}$ on average. This tendency to move to the right corresponds to the distribution of the coins, as more coins were located on the right side of the field. The importance of this finding is discussed in the next section. See also Figure 7.

Next, we turn to the vertical movement in the game, which was due to the flapping and holding the elbows close to the body. In Figure 8, we show the movement of elbows. We calculated the distance between the two elbows and 


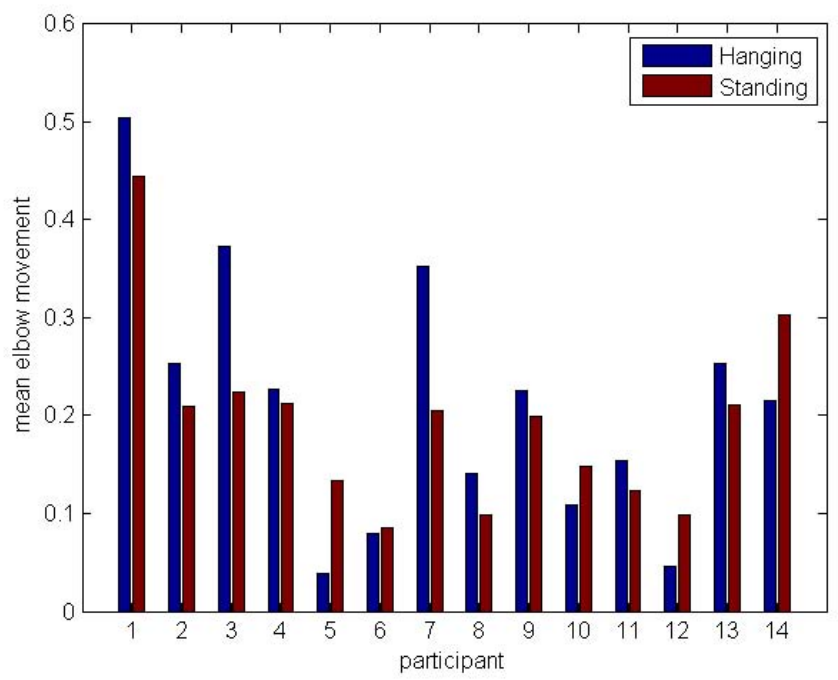

Fig. 8. Bar plot showing mean movement of elbow distance in mm over time

took the difference between successfully recognized frames. This is directly related to the actual recognized elbow joints and to the intention to flap. There is no significant difference in the mean amount of movement between the two conditions.

Open-ended Interview. Five participants clearly indicated that they preferred the hanging over the standing condition. For instance, one participant stated "Hanging was much more fun, the other one is not so special" and some of them stated they wanted to play the hanging game again, or that the session was too short. The other participants were not as open about their preference. Interestingly, even though the players incurred physical discomfort in the hanging position, no one indicated they preferred the standing position. Apart from their preference, four participants indicated the control or recognition had to be improved, e.g. "The translation is a little bit unnatural, was expecting to be more like a child flying". This was due to the fact that instead of stepping to the sides to make the avatar go to any one side, he thought it would be better to rotate his upper body to do so.

\section{Discussion and Future Work}

We have implemented Hang in There, a novel body-centric installation that aims to encourage physical exertion and an enhanced sense of immersion in the player. To this end, we designed an installation where the user is suspended on a climbing rope while playing a coin-collecting game. The player's avatar is controlled using body movements. We wanted to research how body position and movement could 
affect the engagement of the player. The interactive playground was tested with fourteen participants that each played the game in two conditions: in a normal standing position and hanging in a forward tilted position.

Our results do not show a statistical significant difference in engagement between the two conditions, although there appeared to be a tendency to be more engaged in the hanging condition. Testing more participants could prove whether this difference is significant. Several participants indicated they liked the experience of hanging more. When asked if they would like to play the game again, they were more inclined to play in the hanging position, approaching significance on a corrected confidence level. The hanging condition did not lead to an increased amount of body movement. In fact, there was less arm movement in the hanging condition. One possible explanation of this observation is that keeping the body upright in the hanging condition required exertion. With the current measurements, we are not able to investigate this into more detail. Measuring muscle activations might provide the data to perform these analyses.

An important finding came from the displacement in the horizontal direction of the players. Since we wanted the players to move sideways instead of staying in the center of the field, we located the majority of the coins on the sides. Moreover, there were more coins to the right of the field than to the left. We found that, in both conditions, the players were moving to the right more often than to the left. This shows that it would be possible to modify how people play a game, or persuade them to play in a specific manner, by changing the elements of the game and their disposition. Instead of scattering the coins all over the field, in a future version we might place them in other patterns, to stimulate the players to follow these patterns in their movement. Having a trail of coins that crosses the field might be better, since the players would have to move more in order to score.

Based on the positive feedback of the participants, we think our installation has potential for further development. The action recognition, control of the game and comfort could be improved, to increase the enjoyment of playing the game. To reduce the delay between body movement and in-game response, induced both by the Kinect recognition software and the game's working principles, we will consider more direct measurements. One solution could be to use a Wii-controller or accelerometer attached to the upper arm for recognition of flapping. A solution that is considered for detecting movements to the sides, is the use of less demanding computer vision algorithms based on the depth channel of the Kinect. Furthermore, new versions of the Kinect sensor might also lead to a reduction of this delay.

Given the close relation between engagement and social play, we expect that the game can be more enjoyable, but also lead to more exertion, when played with two players. Future work will be aimed at investigating how competition and cooperation can be achieved, and how this impacts both the engagement and level of exertion. To this end, two players could be suspended from a rope, sideby-side. Even though each player has their own side of the platform, interesting interactions could take place in the center of the platform. For instance, players 
holding hands to remain in the center to avoid obstacles, or pushing each other to the sides when competing for resources in this shared physical space. In such a scenario players are not required to fixate solely on the projection to keep performing in the game. These kind of interactions could allow for a further increase of social play [1].

Moreover, we consider improving the immersion of the game by 3D projections in combination with 3D glasses. To further add to an increased sense of flying, future work could include fans, more realistic sound effects and 3D sound localization.

Finally, we expect that the Hang in There installation provides a suitable platform for the research into exertion and engagement. We plan to investigate more closely how certain aspects of the game play such as the control and body position affect the level and type of exertion. Measurements of muscle contraction and more accurate body movement could help in shining a light on this topic.

Acknowledgments. This publication was supported by the Dutch national program COMMIT. The authors would like to thank Yves Rybarczyk and Tiago Cardoso for organizing eNTERFACE 2013, of which the project "Body-Centric Play" was part of. We would also like to thank Dirk Heylen for his support and Jan Kolkmeijer for sharing his expertise of Unity 3D.

\section{References}

1. Bianchi-Berthouze, N.: Understanding the role of body movement in player engagement. Human-Computer Interaction 28(1), 40-75 (2013)

2. Bianchi-Berthouze, N., Kim, W.W., Patel, D.: Does body movement engage you more in digital game play? and why? In: Paiva, A.C.R., Prada, R., Picard, R.W. (eds.) ACII 2007. LNCS, vol. 4738, pp. 102-113. Springer, Heidelberg (2007)

3. Chen, M., Kolko, B.E., Cuddihy, E., Medina, E.: Modeling but NOT measuring engagement in computer games. In: Proceedings of the International Conference on Games + Learning + Society Conference, pp. 55-63. Madison, WI (2011)

4. Fels, S., Yohanan, S., Takahashi, S., Kinoshita, Y., Funahashi, K., Takama, Y., Chen, G.T.-P.: User experiences with a virtual swimming interface exhibit. In: Kishino, F., Kitamura, Y., Kato, H., Nagata, N. (eds.) ICEC 2005. LNCS, vol. 3711, pp. 433-444. Springer, Heidelberg (2005)

5. Finkelstein, S., Nickel, A., Lipps, Z., Barnes, T., Wartell, Z., Suma, E.A.: Astrojumper: Motivating exercise with an immer sive virtual reality exergame. Presence: Teleoperators and Virtual Environments 20(1), 78-92 (2011)

6. Hämäläinen, P., Ilmonen, T., Höysniemi, J., Lindholm, M., Nykänen, A.: Martial arts in artificial reality. In: Proceedings of the International Conference on Human Factors in Computing Systems (CHI), Portland, OR, pp. 781-790 (2005)

7. Hillier, A.: Childhood overweight and the built environment: Making technology part of the solution rather than part of the problem. The Annals of the American Academy of Political and Social Science 615(1), 56-82 (2008)

8. Lindley, S.E., Le Couteur, J., Berthouze, N.: Stirring up experience through movement in game play: Effects on engagement and social behaviour. In: Proceedings of the International Conference on Human Factors in Computing Systems (CHI), Florence, Italy, pp. 511-514 (2008) 
9. Moreno, A., van Delden, R., Poppe, R., Reidsma, D.: Socially aware interactive playgrounds. Pervasive Computing 12(3), 40-47 (2013)

10. Mueller, F., Agamanolis, S., Picard, R.: Exertion interfaces: sports over a distance for social bonding and fun. In: Proceedings of the International Conference on Human Factors in Computing Systems (CHI), Fort Lauderdale, FL, pp. 561-568 (2003)

11. Mueller, F., Bianchi-Berthouze, N.: Evaluating exertion games. In: Evaluating User Experience in Games. Human-Computer Interaction Series, pp. 187-207. Springer (2010)

12. Mueller, F., Gibbs, M.R., Vetere, F.: Design influence on social play in distributed exertion games. In: Proceedings of the International Conference on Human Factors in Computing Systems (CHI), Boston, MA, pp. 1539-1548 (2009)

13. Mueller, F., Toprak, C., Graether, E., Walmink, W., Bongers, B., van den Hoven, E.: Hanging off a bar. In: Extended Abstracts of the International Conference on Human Factors in Computing Systems (CHI), Austin, TX, pp. 1055-1058 (2012)

14. Nijholt, A., Pasch, M., van Dijk, B., Reidsma, D., Heylen, D.: Observations on Experience and Flow in Movement-Based Interaction. In: Whole Body Interaction. Human-Computer Interaction Series, pp. 101-119. Springer (2011)

15. Pasch, M., Bianchi-Berthouze, N., van Dijk, B., Nijholt, A.: Movement-based sports video games: Investigating motivation and gaming experience. Entertainment Computing 1(2), 49-61 (2009)

16. Poppe, R., van Delden, R., Moreno, A., Reidsma, D.: Interactive Playgrounds for Children. In: Playful Interfaces: Interfaces that Invite Social and Physical Interaction. Springer (to appear)

17. Riskind, J.H., Gotay, C.C.: Physical posture: Could it have regulatory or feedback effects on motivation and emotion? Motivation and Emotion 6(3), 273-298 (1982)

18. Soares, L.P., Nomura, L., Cabral, M.C., Nagamura, M., Lopes, R.D., Zuffo, M.K.: Virtual hang-gliding over rio de janeiro. In: ACM SIGGRAPH 2005 Emerging Technologies, Los Angeles, California, p. 29 (2005)

19. Soler-Adillon, J., Parés, N.: Interactive slide: An interactive playground to promote physical activity and socialization of children. In: Proceedings of the International Conference on Human Factors in Computing Systems (CHI), Boston, MA, pp. 2407-2416 (2009)

20. Tetteroo, D., Reidsma, D., van Dijk, E., Nijholt, A.: Design of an interactive playground based on traditional children's play. In: Proceedings of the International Conference on Intelligent Technologies for Interactive Entertainment (INTETAIN), Genova, Italy, pp. 129-138 (2011)

21. van Delden, R., Reidsma, D.: Meaning in life as a source of entertainment. In: Reidsma, D., Katayose, H., Nijholt, A. (eds.) ACE 2013. LNCS, vol. 8253, pp. 403-414. Springer, Heidelberg (2013)

22. Vandewater, E.A., Shim, M.S., Caplovitz, A.G.: Linking obesity and activity level with children's television and video game use. Journal of Adolescence 27(1), 71-85 (2004)

23. Yao, L., Dasgupta, S., Cheng, N., Spingarn-Koff, J., Rudakevych, O., Ishii, H.: RopePlus: Bridging distances with social and kinesthetic rope games. In: Proceedings of the International Conference on Human Factors in Computing Systems (CHI), Vancouver, Canada, pp. 223-232 (2011) 


\section{A Gaming Engagement Questionnaire Revised (GEQ-R)}

1. Were you able to anticipate what would happen next in response to the actions you initiated?*

$\begin{array}{lllllll}1 & 2 & 3 & 4 & 5 & 6 & 7\end{array}$

Notatall $\odot \circ \bigcirc \bigcirc \bigcirc \bigcirc$ Completely

2 - How much delay did you experience between your actions and the expected outcomes within the game?*

$$
\begin{array}{lllllll}
1 & 2 & 3 & 4 & 5 & 6 & 7
\end{array}
$$

Long delay 000000 No delays

3. How appropriate were the physical movements for the game? *

$\begin{array}{lllllll}1 & 2 & 3 & 4 & 5 & 6 & 7\end{array}$

Not appropriate $\odot \odot \bigcirc \bigcirc \bigcirc$ Very appropriate

4. How well were you able to understand the physical movements for the game? *

$\begin{array}{lllllll}1 & 2 & 3 & 4 & 5 & 6 & 7\end{array}$

Notatall $\bigcirc \circ \bigcirc \bigcirc \bigcirc \bigcirc$ Completely

5. How natural did you find the physical movements for the game? *

$\begin{array}{lllllll}1 & 2 & 3 & 4 & 5 & 6 & 7\end{array}$

Not natural $\odot \circ \circ \bigcirc \circ \bigcirc \bigcirc$ Very natural

6. How appropriate was the graphical interface for the game? *

$\begin{array}{lllllll}1 & 2 & 3 & 4 & 5 & 6 & 7\end{array}$

Not appropriate $\odot \odot \bigcirc \bigcirc \bigcirc \bigcirc$ Very appropriate

7. How well were you able to understand the graphical interface for the game? *

$\begin{array}{lllllll}1 & 2 & 3 & 4 & 5 & 6 & 7\end{array}$

Notatall $\odot \odot \bigcirc \bigcirc \bigcirc \bigcirc$ Completely

8. How proficient at controlling the game did you feel at the end of today's gaming session? *

$\begin{array}{lllllll}1 & 2 & 3 & 4 & 5 & 6 & 7\end{array}$

Not proficient $\odot \odot \odot \odot \odot$ Very proficient 
9. How well were you able to identify what game pieces/objects/models represented? *

$\begin{array}{lllllll}1 & 2 & 3 & 4 & 5 & 6 & 7\end{array}$

Notatall $\odot \odot \odot \odot \odot \bigcirc$ Completely

10. How consistent were the graphics and controls together?*

$\begin{array}{lllllll}1 & 2 & 3 & 4 & 5 & 6 & 7\end{array}$

Not consistent $\odot \odot \odot \odot \odot \odot$ Very consistent

11. How involved were you in the game experience? *

$\begin{array}{lllllll}1 & 2 & 3 & 4 & 5 & 6 & 7\end{array}$

Not involved $\odot \odot \odot$ Fully involved

12. Were you involved in the game to the extent that you lost track of time? *

$\begin{array}{lllllll}1 & 2 & 3 & 4 & 5 & 6 & 7\end{array}$

Notatall $\odot \odot \odot \bigcirc \bigcirc \bigcirc \bigcirc$ Completely

13. How much did you feel like you were inside the game world? *

$\begin{array}{lllllll}1 & 2 & 3 & 4 & 5 & 6 & 7\end{array}$

Notatall $\odot \odot \bigcirc \bigcirc \bigcirc \bigcirc \bigcirc$ Completely

14. How often do you play other games of this genre? *

$\begin{array}{lllllll}1 & 2 & 3 & 4 & 5 & 6 & 7\end{array}$

Never $\odot \circ \circ \odot \circ \circ$ Often

15. How enjoyable do you find the content and theme of this game? *

$\begin{array}{lllllll}1 & 2 & 3 & 4 & 5 & 6 & 7\end{array}$

Not enjoyable $\odot \odot \odot$ Very enjoyable

16. How interested are you in playing this game again? *

$\begin{array}{lllllll}1 & 2 & 3 & 4 & 5 & 6 & 7\end{array}$

Not interested $\odot \odot \bigcirc$

17. How much did the users's interface interfere with your ability to perform actions within the game? *

$\begin{array}{lllllll}1 & 2 & 3 & 4 & 5 & 6 & 7\end{array}$

Notatall $\bigcirc \odot \odot \bigcirc \bigcirc$

18. To what extent did you feel spatially disoriented with your ability to perform actions within the game?*

$\begin{array}{lllllll}1 & 2 & 3 & 4 & 5 & 6 & 7\end{array}$

Notatall $\odot \odot \odot \bigcirc \bigcirc$ Very much

19. To what extent are you interested in engaging in further exploration of the game's environment? *

$\begin{array}{lllllll}1 & 2 & 3 & 4 & 5 & 6 & 7\end{array}$

Notinterested $\bigcirc \odot \bigcirc \bigcirc \bigcirc \bigcirc \bigcirc$ Very interested 
20. How completely were you engaged in the game?*

$\begin{array}{lllllll}1 & 2 & 3 & 4 & 5 & 6 & 7\end{array}$

Not engaged $\bigcirc 0000$ Completely engaged

21. To what extent did events such as noise occurring outside of the game distract you from playing the game?*

$$
\begin{array}{lllllll}
1 & 2 & 3 & 4 & 5 & 6 & 7
\end{array}
$$

Not at all $\bigcirc \bigcirc \bigcirc \bigcirc \bigcirc$ Very much

22. To what extent did the discomfort of your position distract you from playing the game? *

$\begin{array}{lllllll}1 & 2 & 3 & 4 & 5 & 6 & 7\end{array}$

Notatall $\odot \odot \odot \bigcirc \bigcirc \odot$ Very much 